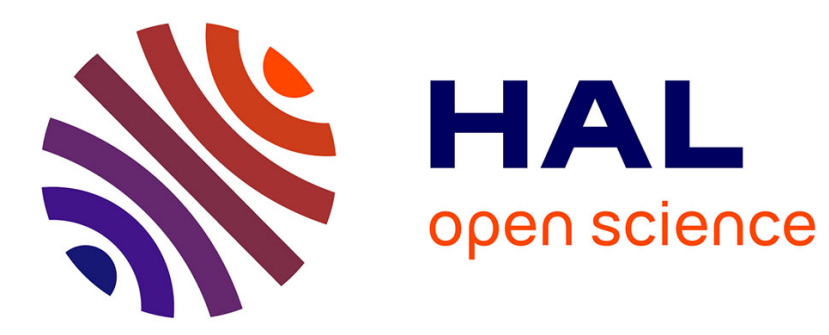

\title{
In Vivo FRET Imaging to Predict the Risk Associated with Hepatic Accumulation of Squalene-Based Prodrug Nanoparticles.
}

Fanny Cayre, Simona Mura, Bohdan Andreiuk, Dunja Sobot, Sandrine Gouazou, Didier Desmaele, Andrey Klymchenko, Patrick Couvreur

\section{To cite this version:}

Fanny Cayre, Simona Mura, Bohdan Andreiuk, Dunja Sobot, Sandrine Gouazou, et al.. In Vivo FRET Imaging to Predict the Risk Associated with Hepatic Accumulation of Squalene-Based Prodrug Nanoparticles.. Advanced healthcare materials, 2017, 7 (3), pp.1700830. 10.1002/adhm.201700830 . hal-02347607

\section{HAL Id: hal-02347607 https://hal.science/hal-02347607}

Submitted on 27 Nov 2019

HAL is a multi-disciplinary open access archive for the deposit and dissemination of scientific research documents, whether they are published or not. The documents may come from teaching and research institutions in France or abroad, or from public or private research centers.
L'archive ouverte pluridisciplinaire HAL, est destinée au dépôt et à la diffusion de documents scientifiques de niveau recherche, publiés ou non, émanant des établissements d'enseignement et de recherche français ou étrangers, des laboratoires publics ou privés. 
In Vivo FRET Imaging to Predict the Risk Associated with Hepatic Accumulation of Squalene-Based Prodrug Nanoparticles

Fanny Cayre, Simona Mura*, Bohdan Andreiuk, Dunja Sobot, Sandrine Gouazou, Didier Desmaele, Andrey S. Klymchenko, Patrick Couvreur*

F. Cayre, Dr. S. Mura, Dr. D. Sobot, S. Gouazou, Dr. D. Desmaele, Pr. P. Couvreur.

Institut Galien Paris-Sud, UMR 8612, CNRS, Univ Paris-Sud, Université Paris-Saclay, Faculté de Pharmacie, 5 rue Jean-Baptiste Clément, F-92296 Châtenay-Malabry cedex, France.

E-mail: simona.mura@u-psud.fr; patrick.couvreur@u-psud.fr

B. Andreiuk.

(i) Laboratoire de Biophotonique et Pharmacologie, UMR CNRS 7213, University of Strasbourg, 74 route du Rhin, 67401 Illkirch Cedex, France, (ii) Organic Chemistry Department, Chemistry Faculty, Taras Shevchenko National University of Kyiv, 01601 Kyiv, Ukraine

Dr A.S. Klymchenko

Laboratoire de Biophotonique et Pharmacologie, UMR CNRS 7213, University of Strasbourg, 74 route du Rhin, 67401 Illkirch Cedex, France

Keywords: FRET, prodrug nanoparticles, in vivo non-invasive imaging 


\begin{abstract}
Förster resonance energy transfer (FRET) is used here for the first time to monitor the in vivo fate of nanoparticles made of the squalene-gemcitabine prodrug and two novel derivatives of squalene with the cyanine dyes 5.5 and 7.5 , which behave as efficient FRET pair in the NIR region. Following intravenous administration, nanoparticles initially accumulate in the liver, then they show loss of their integrity within $2 \mathrm{~h}$ and clearance of the squalene bioconjugates is observed within $24 \mathrm{~h}$. Such awareness is a key prerequisite before introduction into clinical settings.
\end{abstract}


The use of nanotechnology-based medicines (i.e., nanomedicines) for drug delivery holds the potential to overcome limits associated to conventional chemotherapies (e.g., non-specific biodistribution, metabolization, rapid excretion), thus providing a solution to various medical challenges. $^{[1]}$ Although the major advances have been done in the field of cancer, nanomedicines may also be beneficial in several other therapeutic areas by providing an increase in the drug therapeutic index. ${ }^{[2]}$ Generally, drugs are physically encapsulated into the nanocarrier, but the design of prodrug-based nanomedicines has also emerged as alternative strategy. This approach enabled to face drawbacks such as $(i)$ the low drug loading generally achieved with physical encapsulation processes and (ii) the uncontrolled drug release (i.e., "burst release"), corresponding to that fraction of the drug only adsorbed onto the nanocarrier surface. $^{[3]}$

In this context, terpene-based prodrug nanoparticles (NPs) have been proposed by our group a few years ago. ${ }^{[4]}$ Among them, the biocompatibility of squalene (SQ) (a natural lipid, intermediate in the cholesterol biosynthesis) promoted its large investigation as drug carrier, and allowed the design of a small library of bioconjugates by covalent conjugation of various drug molecules to squalene (i.e., the so-called "squalenoylation approach"). ${ }^{[5]}$ All these bioconjugates demonstrated the capacity to spontaneously self-assemble in aqueous solution as nanoparticles, without requiring any other carrier material. The most extensive investigation has been performed with nanoparticles made of the bioconjugate in which the squalene is linked to gemcitabine (SQGem) (Figure 1), a nucleoside analogue clinically indicated in the treatment of various solid tumors. Despite promising results in a series of experimental tumor models, ${ }^{[6]}$ any further step toward a clinical investigation of these SQGem NPs has been hindered, up to now, by the lack of clear knowledge on their fate after intravenous administration. In particular, the appreciation of liver accumulation, the major organ of nanoparticles distribution, is of outmost importance before the first human use. 
Indeed liver overload of nanoparticulate material may induce insurgence of severe liver injury and irreversible impairment of its functionality. ${ }^{[7]}$

In order to face this issue, we have monitored in this study the in vivo fate of SQGem NPs taking advantage of the Förster resonance energy transfer (FRET) principle, a non-radiative process, which relies on the transfer of energy from an excited molecule (i.e., donor, D) to an acceptor one (i.e., acceptor, A) closely located. ${ }^{[8]}$ The strong dependency of FRET from the distance between the FRET pair $(1-10 \mathrm{~nm})$ has made this technique extremely appealing to monitor biological phenomena, ${ }^{[9]}$ integrity of nanoparticles ${ }^{[10]}$ and micelles, ${ }^{[11]}$ but to our knowledge, it had not found application yet to follow the in vivo integrity of nanomedicines with an already demonstrated therapeutic efficacy. To reach this goal two new lipophilic 5.5 and 7.5 cyanine dyes bearing a squalene appendage tethered to the benzo[e]indol- 3 - ium moiety through a six-carbon linker have been synthetized (SQCy5.5 and SQCy7.5) (Figure 1). First, following established protocols for the synthesis of asymmetrically substituted cyanines, ${ }^{[12]}$ we prepared Cy5.5 and Cy7.5 dyes bearing a single alcohol group. Then, esterification with squalenic acid allowed to obtain the final conjugates SQCy5.5 and SQCy7.5 (Scheme 1, Supporting Information). The emission spectrum of the SQCy5.5 overlaps the absorption spectrum of the SQCy7.5 (Figure S13, Supporting Information), making them an ideal donor/acceptor FRET pair. In addition, the emission spectra of the two dyes are perfectly separated, and both emit in the so called "biological transparency NIR window " (i.e., NIR-I, 700-900 nm). ${ }^{[13]}$ Offering the opportunity to overcome the problems associated to tissue scattering and absorption observed at lower wavelengths (i.e., visible spectrum), these dyes can be efficiently applied for non-invasive in vivo imaging on preclinical animal models. 

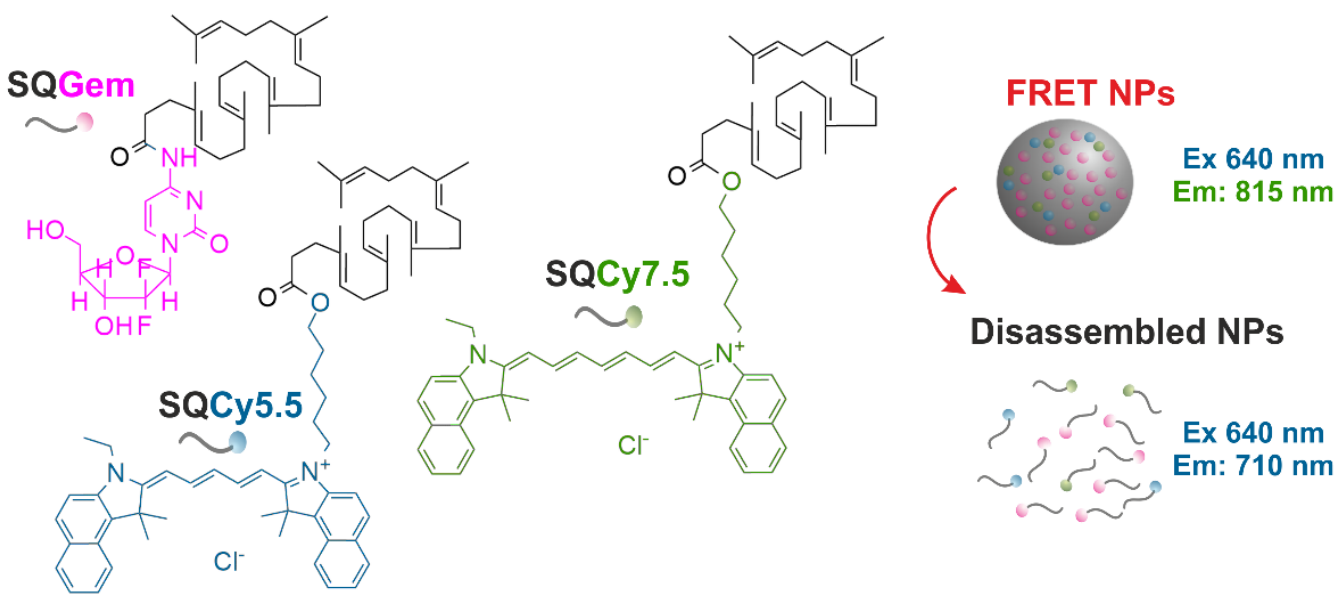

Figure 1. Structures of squalene bioconjugates used for the formulation of FRET NPs and schematic representation of the use of FRET signal to monitor NPs integrity status.

Fluorescent FRET NPs have been prepared by nanoprecipitation in water of an ethanolic solution of SQGem with the two dyes (i.e., SQCy5.5 and SQCy7.5), followed by evaporation of the organic solvent under vacuum. While keeping constant the amount of SQGem (2 mg $\mathrm{mL}^{-1}$ ), optimization of the formulation was performed by tuning the weight percentage of the dyes with respect to the SQGem moiety. As displayed in Figure 2a, the emission signal of the acceptor (SQCy7.5) collected after excitation of the donor (SQCy5.5) at $640 \mathrm{~nm}$ increased with the total content of the dyes (weight SQdye/weight SQgem) in the formulation. Dilution of NPs in organic solvent was used to mimic the complete disassembly of the NPs. It resulted in disappearance of the SQCy7.5 emission and emergence of the strong SQCy5.5 signal (Figure 2b), which was proportional to the dye concentration. This indicated that the energy transfer signal could be observed only when NPs remained intact with the two dyes at a close distance. Accordingly, this FRET pair could be used to monitor the integrity status of the 
nanoparticles. The highest FRET signal was obtained for the nanoformulation with the highest dye content $(0.6 \%$ w/w of each). This formulation, from now indicated as FRET NPs, has thus been selected for all following studies. The loading of both dyes in the FRET NPs led to an overall quantum yield of $8.4 \%$, (Figure S14, Supporting Information) which was close to the value measured for FRET nanoemulsion droplets encapsulating comparable dyes. ${ }^{[10 a]}$ Although lower compared to the value measured following dilution with ethanol (that is the donor quantum yield, $21.2 \%$ ), this quantum yield assured that the brightness of the FRET NPs was high enough to monitor, in real time, the NPs integrity following their intravenous administration.

NPs containing 0.6\% w/w of SQCy5.5 (Donor NPs, D-NPs) or SQCy7.5 (Acceptor NPs, ANPs) only, were prepared as described above and used as controls (Figure S15, Supporting Information). The common squalene unit in SQGem, SQCy5.5 and SQCy7.5 allowed the selfassembly of mixed bioconjugates as nanoparticles with a mean diameter of $139 \mathrm{~nm}$, a narrow size distribution and a comparable surface charge $(\sim-45 \mathrm{mV})$ (Table S1, Supporting Information). To mimic different percentages of FRET NPs integrity (expressed as \% of intact FRET NPs with respect to the total dye amount in the mixture), various proportions of FRET NPs, D-NPs and A-NPs were blended, keeping constant the overall amount of dyes. As expected, a decrease in FRET NPs integrity \%, was associated to a concentration dependent increase in the donor signal following excitation at $640 \mathrm{~nm}$, (Figure S16, Supporting Information) which became similar to that of the D-NPs alone for the mixture corresponding to $0 \%$ integrity of FRET NPs (Figure S15b,c, Supporting Information). 

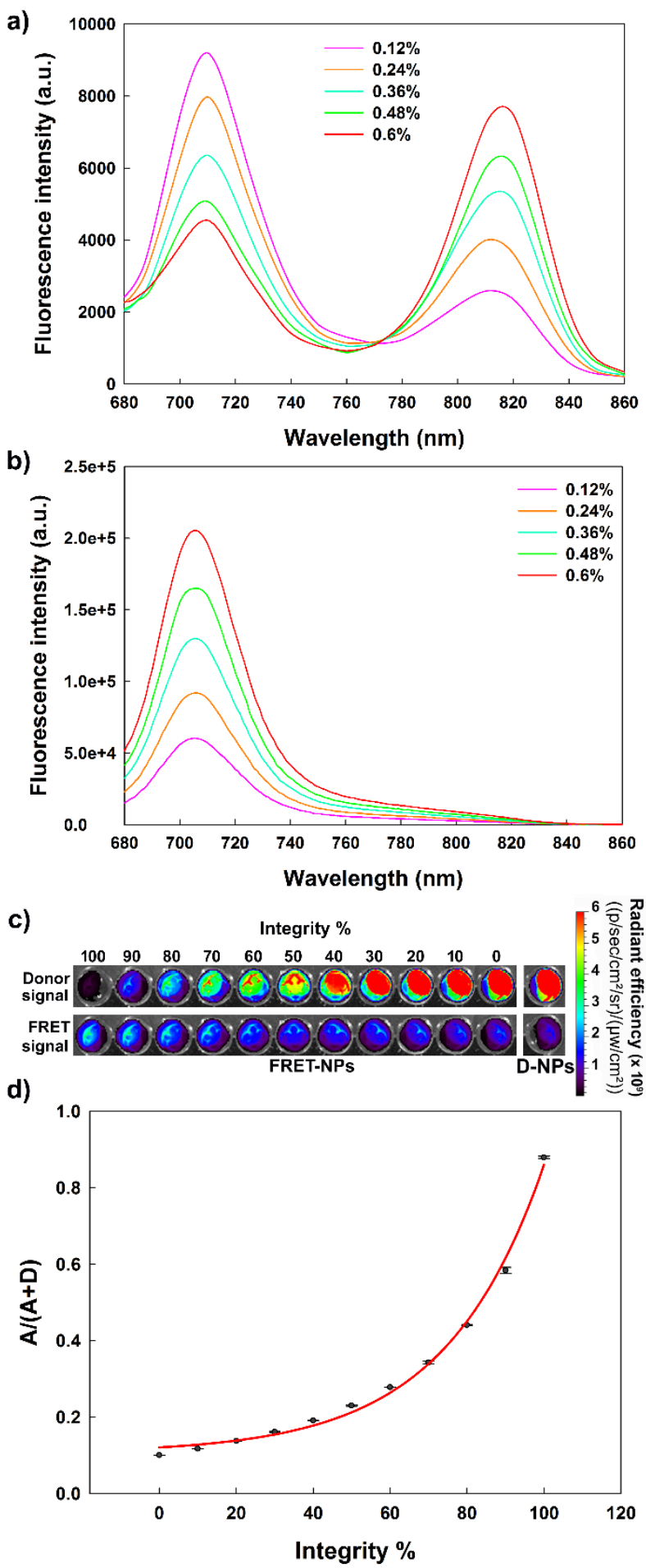

Figure 2. Fluorescence emission spectra (excitation at $640 \mathrm{~nm}$ ) of NPs formulated by tuning the $\%$ of dyes after dilution with (a) water or (b) ethanol. Each formulation contains the same amount of SQCy5.5 and SQCy7. The percentage of each dye with respect to the SQGem moiety is indicated in the figure legend. (c) Images of mixtures of FRET NPs mimicking different integrity \% (excitation at $640 \mathrm{~nm}$ (emission collected at 695-770 nm (donor signal) 
and 810-875 nm (FRET signal)). (d) Calibration curve A/(A+D) ratio vs NP integrity \%. Data represent mean \pm standard deviation $(\mathrm{SD})(\mathrm{n}=2)$.

This behavior was then confirmed by imaging the NPs mixtures using an in vivo imaging system (IVIS ${ }^{\circledR}$, PerkinElmer) after excitation at $640 \mathrm{~nm}$ (Figure 2c). Moving to the lowest \% of FRET NPs integrity, variation of the pseudocolor toward the red (high signal) was observed in the donor emission filter, while the color changed from light blue to dark blue/violet (low values) in the FRET emission one. The image of fully disintegrated NPs (corresponding to a mixture of D-NPs and A-NPs only) matched that of D-NPs only, thus confirming that in the absence of FRET NPs integrity, no FRET signal could be detected. Following signal quantification, the so called FRET proximity ratio (i.e., $\mathrm{A} /(\mathrm{A}+\mathrm{D})){ }^{[14]}$ coinciding with the different FRET NPs integrity \% was then calculated. By plotting this semi-quantitative measurement of the FRET efficiency versus the integrity percentage, it was then possible to construct a calibration curve of the FRET NPs integrity (Figure 2d), useful to assess their stability over time.

This stability was then evaluated at $37^{\circ} \mathrm{C}$ after opportune dilution in (i) water, (ii) murine blood and (iii) ethanol (Figure 3). Dilution in organic solvent was used as a control for complete disintegration of FRET NPs and absence of energy transfer, which corresponded to a baseline value of the $\mathrm{A} /(\mathrm{A}+\mathrm{D})$ ratio (around 0.1 , Figure 3, white bars). In water, no modification of the FRET proximity ratio was detected until $6 \mathrm{~h}$ of incubation, while it progressively decreased after $24 \mathrm{~h}$. Contrarily, a rapid reduction of the FRET efficiency was already observed after 35 minutes of incubation in blood and the value reached the baseline after only $4 \mathrm{~h}$. According to the calibration curve, these results revealed that FRET NPs were still intact in water after $6 \mathrm{~h}$ and that only a minimal disintegration occurred after $24 \mathrm{~h}(84 \pm$ $7 \%$ integrity). Disintegration slowly continued but $60 \%$ of nanoparticles still remained intact 
after $48 \mathrm{~h}$ incubation. On the contrary, the integrity of FRET NPs was only $30 \%$ at $2 \mathrm{~h}$ post incubation in blood. Afterwards, the signal was too low to be quantified. As expected, for DNPs incubated in the same media, the $\mathrm{A} /(\mathrm{A}+\mathrm{D})$ ratio corresponded to the baseline value, thus clearly confirming that the FRET signal originated only from the transfer of energy between the two dyes being at close vicinity within the same nanoparticle (Figure S17a, Supporting Information). The physical mixture of D-NPs and A-NPs showed the same behaviour in water and ethanol, while a slight FRET signal appeared in blood (Figure S17b, Supporting Information). Being the two dyes loaded into separate NPs, such observation might be explained by a partitioning in blood components (e.g., albumin, lipoproteins $)^{[15]}$ with few dye molecules coming close enough to trigger the energy transfer to occur. Nevertheless, the $\mathrm{A} /(\mathrm{A}+\mathrm{D})$ ratio values were low $(\leq 0.2)$ and the phenomenon was only transient.

Once verified that FRET NPs integrity could be monitored following the variation of the FRET efficiency, we applied it to detect in real time the integrity status of the NPs after intravenous administration in mice. In particular, we aimed at elucidating whether these nanoparticles could accumulate into the liver, in which form (intact or disassembled) and for how long, since severe liver injury consequent to hepatic accumulation of NPs represents a major toxicological issue. According, a prediction of the risk associated to the NPs exposure is crucial to shorten the translation of nanomedicines from the bench to the bedside. In this context, the FRET approach could represent a valuable tool to assess the in vivo fate of the SQGem NPs. 


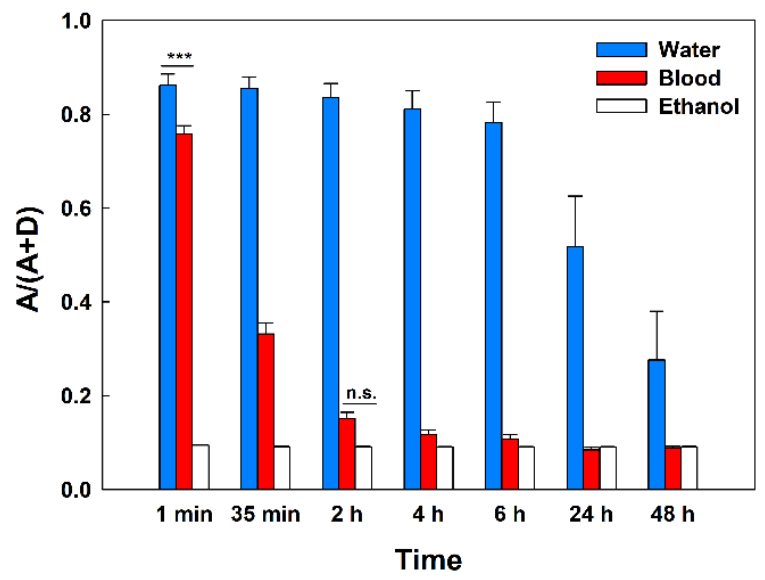

Figure 3. In vitro stability study of FRET NPs at $37^{\circ} \mathrm{C}$ after dilution with (i) MilliQ ${ }^{\circledR}$ water (blue bars), (ii) mouse blood (red bars) or (iii) ethanol (white bars). Results are expressed as mean \pm standard deviation $(\mathrm{SD})(\mathrm{n}=2)$. $(* * *$ indicates $\mathrm{p}<0.001$, n.s. indicates no significant differences, by two-way Anova with a Tukey's multiple comparison post-test).

Thus, mice have been injected with FRET NPs intravenously in the lateral tail vein and then imaged at regular time points. Following excitation at $640 \mathrm{~nm}$, the FRET and Donor signals were collected on the 810-875 $\mathrm{nm}$ (acceptor channel) and 695-775 $\mathrm{nm}$ (donor channel) emission filters, respectively. The first image has been acquired at 35 minutes post injection. Then, the evolution of the signal has been monitored over time (Figure 4). The variation of the pseudocolor in the acquired images suggested a fast accumulation of the FRET NPs into the liver, which reached the maximum at 35 minutes. Importantly, the signal recorded for FRET NPs at the donor channel (Donor signal) was weaker compared to that of the control DNPs, whereas at the acceptor channel (FRET signal) the opposite was observed (Figure 4). This result clearly indicated that at 35 min post injection, FRET NPs preserved at least partially their integrity in the liver. On the contrary, after $2 \mathrm{~h}$, the intensity increase at the donor channel was accompanied by the decrease at the FRET channel (Figure 4), so that the signals at the two channels became similar to those for the control D-NPs at $2 \mathrm{~h}$ (Figure S19, Supporting Information). ${ }^{\phi}$ Thus, FRET NPs rapidly disassembled, leading to an increase in 
the distance between donor and acceptor dyes and consequently a loss of the FRET signal. Then, the progressive decrease of all signals clearly indicated the elimination of the bioconjugates. As expected, according to NPs disintegration and elimination from the liver, the acceptor signal (collected on the 810-875 $\mathrm{nm}$ emission filter following excitation at 745 $\mathrm{nm}$ ) displayed a progressive decrease over time too (Figure S18, Supporting Information). Similar signal evolution was observed after injection of D-NPs and A-NPs alone (Figure S19 and S20, Supporting Information). Control mice displayed negligible background signal (Figure S21, Supporting Information).

Signal has been then quantified by tracing a region of interest at the level of the liver. In agreement with the acquired images, the signal collected at the donor channel, progressively increased, reaching the highest intensity at $2 \mathrm{~h}$, and then decreased (solid green line) (Figure S22a, Supporting Information). No quantification has been possible after $6 \mathrm{~h}$. On the contrary, for the FRET signal collected at the acceptor channel, the maximum value corresponded to the first acquisition at 35 minutes and then a reduction of the signal was measured (solid red line). D-NPs showed an analogous behaviour (green dashed line) (Figure S22a, Supporting Information). 


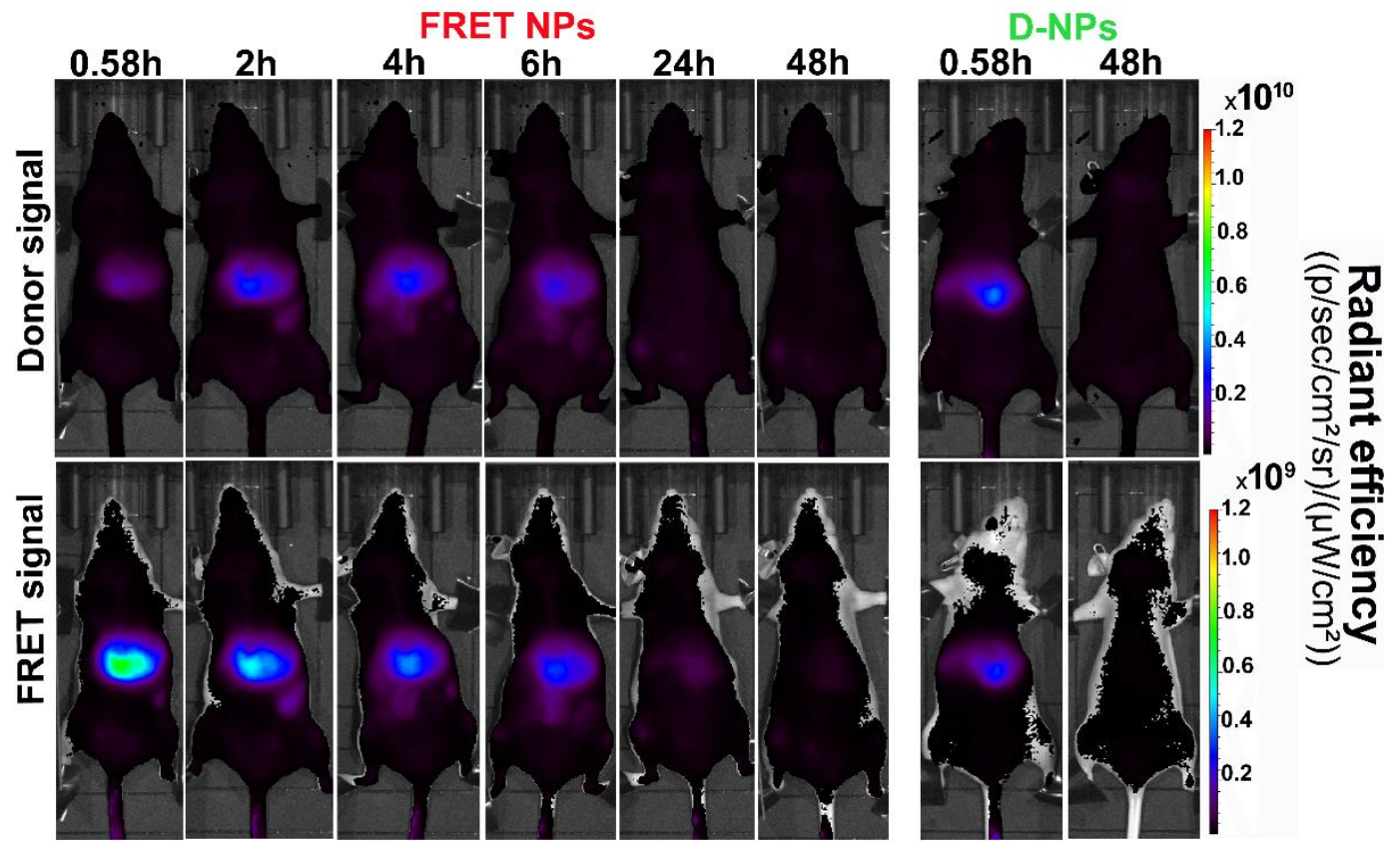

Figure 4. In vivo representative images of one mouse after intravenous injection of FRET NPs or D-NPs Excitation at $640 \mathrm{~nm}$ and emission collection at $695-770 \mathrm{~nm}$ (donor signal) and 810-875 nm (FRET signal).

These results clearly demonstrated that SQGem NPs disassembled in the liver, as shown by FRET efficiency, (Figure S22b, Supporting Information) which peaked at 35 minutes (maximal accumulation of intact nanoparticles) and then dropped down after $2 \mathrm{~h}$ post injection. No FRET efficiency was measured following injection of D-NPs, thus confirming, once again, that the FRET signal originated only from the transfer of energy between the pair of FRET dyes into the same nanoparticle. According to these data, the integrity of the FRET NPs in the liver followed a kinetic similar to that observed in vitro in the blood, with around $56 \%$ of intact NPs 35 minutes after injection and then a fast disintegration, leading to less than $10 \%$ of NPs integrity at $2 \mathrm{~h}$ post administration. It has to be noted that only scarce information is instead available about long-term liver accumulation of biodegradable polymer NPs (e.g. poly(lactic-co-glycolic acid) (PLGA) NPs) despite their broad use in the drug delivery field. Indeed, most of the studies evaluated only a few hours post-administration, and the 
monitoring mainly relied on the quantification of physically encapsulated drugs or fluorescent dyes. Thus, in case of dye (or drug) leakage, detection doesn't sign necessarily the presence of intact or degraded nanoparticles. ${ }^{[16]}$ Regarding longer-term studies, a quantitative analysis of PLGA NPs accumulation and in vivo degradation, ${ }^{[17]}$ revealed that around $10 \%$ and $20 \%$ of the injected dose of respectively $200 \mathrm{~nm}$ and $500 \mathrm{~nm}$ NPs, was still present in the liver 7 days after injection. Moreover, silica and metal nanoparticles were intensively investigated and their long-term persistence raised concerns about potential chronic toxicity. ${ }^{[7,18]}$ For instance after intravenous injection of silica NPs ${ }^{[19]}$ and gold NPs ${ }^{[20]}$ prolonged hepatic retention was observed for up to 1 and 2 months, respectively. CdSeTe quantum dots persisted in the liver for up to six months, ${ }^{[21]}$ while gold and superparamagnetic iron were detected even at 1 year post administration of iron oxide-coated gold nanoparticles. ${ }^{[22]}$

In conclusion, the present study shows that the herein synthesized SQ-based FRET dyes allowed to shed light on the integrity of SQGem NPs after systemic administration. Results unambiguously revealed that these NPs could at least partially preserve their integrity at the initial accumulation in the liver. However, no persistent accumulation of NPs in the hepatic tissue was observed thus suggesting appropriate safety profile and absence of hepatic thesaurismoses, a frequent toxicological event in nanomedicine. This is in agreement with previous reports, where neither alterations of the of hepatic enzymes levels nor morphological damages were observed after intravenous administration of squalene-based NPs. ${ }^{[6 b, 23]}$ On the whole these results would facilitate the future introduction of squalene-based nanomedicines into clinical settings. It has to be noted that application of these dyes to whole-body imaging in humans is still limited by the depth of penetration of the NIR-I light (less than $1 \mathrm{~cm}) .{ }^{[24]}$ However, the development of new dyes, which emit at longer wavelengths (NIR-II region 1000-1700 nm) in combination with advanced instruments endowed with higher sensitivity and resolution, is expected to allow deeper tissue penetration, in the near future. 


\section{Experimental Section}

Materials: Gemcitabine (2',2'-difluorodeoxycitidine) hydrochloride was purchased from Sequoia Research Products Ltd. (Pangbourne, UK). 4-(N)-trinorsqualenoyl-gemcitabine (SQGem) was synthesized as previously described. ${ }^{[4 a]}$ Reagents for the synthesis were purchased from Sigma Aldrich (France) and TCI Chemicals (France) and used without further purification. Analytical grade solvents were purchased from VWR Chemicals (France) or Sigma Aldrich (France). Ultrapure water was obtained with the MilliQ ${ }^{\circledR}$ purification system (Merck Millipore, France). NMR spectra were recorded at $20^{\circ} \mathrm{C}$ on Bruker Avance III 400 $\mathrm{MHz}$ and Bruker Avance III $500 \mathrm{MHz}$ spectrometers. Mass spectra were obtained using an Agilent Q-TOF 6520 mass spectrometer. Flash chromatography was performed on PuriFlash 430 (Interchim Inc).

Formulation and characterisation of fluorescent SQGem nanoparticles (NPS): Fluorescent SQGem NPs were prepared according to the nanoprecipitation technique. Practically, a mixture of SQGem (4 mg mL ${ }^{-1}$ in ethanol), SQCy5.5 and SQCy7.5 (both at $0.5 \mathrm{mg} \mathrm{mL}^{-1}$ in ethanol) was added dropwise into MilliQ ${ }^{\circledR}$ water under magnetic stirring (ethanol/water 0.5:1 v/v). After solvent evaporation under vacuum (Rotavapor ${ }^{\circledR}$ Buchi, France), an aqueous suspension of fluorescent NPs was obtained (final SQGem concentration: $2 \mathrm{mg} \mathrm{mL} \mathrm{m}^{-1}$ ). Keeping constant the concentration of SQGem, different formulations have been prepared by tuning the weight percentage of the dyes with respect to the SQGem. NPs selected for in vitro and in vivo studies contained $0.6 \%$ by weight of each dye (FRET NPs). Control formulations were prepared as described above using $0.6 \%$ of SQCy5.5 (donor NPs (D-NPs)) and $0.6 \%$ of SQCy7.5 (acceptor NPs (A-NPs)), respectively. Size and polydispersity index of the NPs were determined by dynamic light scattering at $25^{\circ} \mathrm{C}$ (Zetasizer Nano ZS, Malvern Instruments, UK, $173^{\circ}$ scattering angle). NP surface charge was investigated using the same apparatus, by zeta potential measurements at $25{ }^{\circ} \mathrm{C}$ after dilution with $1 \mathrm{mM} \mathrm{NaCl}$ solution 
applying the Smoluchowski equation. All measurements were repeated at least three times. For in vitro and in vivo studies, NPs suspensions were brought to isotonicity using dextrose $(5 \% \mathrm{w} / \mathrm{w})$.

Absorbance and fluorescence spectra: Absorbance spectra were measured with a UV-visible spectrophotometer (Lambda 25, Perkin Elmer, USA). Fluorescence emission spectra were measured with a spectrofluorometer (SPEX1681, Horiba, Japan) with an excitation wavelength of $640 \mathrm{~nm}$ (donor) or $760 \mathrm{~nm}$ (acceptor). Measurements were performed after opportune dilution of NPs, which assured an absorbance $\leq 0.1$ at the excitation wavelengths and a total dye concentration $<1 \mu \mathrm{M}$. For relative quantum yield measurement only, the absorption and emission spectra were recorded at RT $\left(25^{\circ} \mathrm{C}\right)$ on a Cary 400 Scan ultravioletvisible spectrophotometer (Varian) and FluoroMax-4 spectrofluorometer (Horiba JobinYvon), respectively. The emission spectra were corrected for the lamp fluctuations and the wavelength-dependent response of the detector. All fluorescence measurements were performed after opportune dilution of NPs (absorbance $\leq 0.1$ ). The excitation wavelength was $630 \mathrm{~nm}$. Quantum yields were calculated using rhodamine 800 in ethanol (quantum yield = $0.25)$ as a reference. ${ }^{[25]}$

Fluorescence imaging settings and image analysis: Images were acquired using the IVIS Lumina $^{\circledR}$ LT Series III (PerkinElmer, USA). After excitation at $640 \mathrm{~nm}$, the donor signal was recovered on the 695-770 $\mathrm{nm}$ emission filter (donor channel) and the FRET signal was recovered on the 810-875 $\mathrm{nm}$ emission filter (acceptor channel). Acceptor signal was recovered on the 810-875 $\mathrm{nm}$ emission filter (acceptor channel) after excitation at $745 \mathrm{~nm}$. Images were processed using the Living Image software (PerkinElmer, USA). For quantification, a region of interest was automatically drawn around $(i)$ the wells of the 96-well 
plates (in vitro studies) or (ii) the mice liver (in vivo studies). Average radiant efficiency values (threshold of 5\% and 30\% for in vitro and in vivo images, respectively) have been used for quantification. The semi-quantitative measurement of the efficiency of the FRET signal (i.e., FRET proximity ratio ${ }^{[14]}$ was calculated as $\mathrm{A} /(\mathrm{A}+\mathrm{D})$ ratio. $\mathrm{A}$ and $\mathrm{D}$ corresponded to the fluorescence intensity of the acceptor (collected on the $810-875 \mathrm{~nm}$ emission filter) and donor (collected on the $695-770 \mathrm{~nm}$ emission filter) respectively, after excitation at $640 \mathrm{~nm}$ (i.e.,donor excitation).

Animals: Four-week-old immunodeficient female mice (athymic nude) were purchased from Envigo Laboratory (France). Animals were housed in an appropriate animal care facility during the experimental period. Mice blood used for in vitro studies was obtained by cardiac puncture under deep terminal anesthesia with pentobarbital, and collected in VACUETTE ${ }^{\circledR}$ EDTA tubes (Greiner Bio-one). Experimental protocols were approved by the Animal Care Committee of the Université Paris-Sud in accordance with the principles of laboratory animal care and the French legislation.

In vitro NPs disaggregation study: In order to mimic the disassembly of FRET NPs, mixtures of FRET NPs (dilution 1:2 with MilliQ ${ }^{\circledR}$ water), D-NPs and A-NPs were prepared. 0\% integrity corresponded to a mixture of D-NPs and A-NPs (ratio 1:1), 50\% integrity corresponded to a mixture of diluted FRET NPs, D-NPs and A-NPs (ratio 0.5:0.25:0.25) and $100 \%$ integrity corresponded to the diluted FRET NPs only. The overall concentration of the dyes was maintained constant. $70 \mu \mathrm{L}$ of each mixture corresponding to different integrity $\%$ were loaded into a 96-well black plate and imaged using the Lumina system. Acquisition has been performed as previously described (see section Fluorescence imaging settings and image 
analysis). Experiment was repeated two times. Results are expressed as mean \pm standard deviation.

Values of $\mathrm{A} /(\mathrm{A}+\mathrm{D})$ ratio were then plotted versus the percentage of NPs integrity and data were fitted (SigmaPlot, Version 12) according to an exponential function (1)

$\mathrm{y}=\mathrm{y}_{0}+\mathrm{ae}^{\mathrm{bx}}$

Where $\mathrm{y}_{0}=0.1055, \mathrm{a}=0.0152$ and $\mathrm{b}=0.0391$.

In vitro stability study: D-NPs and FRET NPs (dilution 1:2 in MilliQ ${ }^{\circledR}$ water) and a D-NPs/ANPs physical mixture (ratio 1:1) were diluted (1:6) with $(i)$ MilliQ $^{\circledR}$ water, (ii) ethanol or (iii) mouse blood and incubated at $37{ }^{\circ} \mathrm{C}$ under stirring over a period of $48 \mathrm{~h}$. Immediately after mixing ( $\mathrm{t}=1$ minute), and then at $\mathrm{t}=35$ minutes $(0.58 \mathrm{~h}), 2 \mathrm{~h}, 4 \mathrm{~h}, 6 \mathrm{~h}, 24 \mathrm{~h}$ and $48 \mathrm{~h}$ post incubation, $70 \mu \mathrm{l}$ of each mixture was loaded in a 96-well black plate (Sigma Aldrich, France) and fluorescence signal was collected with the Lumina system (see section Fluorescence imaging settings and image analysis). The experiment was repeated twice in duplicate. Results are expressed as mean \pm standard deviation.

In vivo non-invasive imaging: $200 \mu \mathrm{L}$ of FRET NPs, D-NPs or A-NPs were administered to athymic mice ( $\mathrm{n}=6$ for FRET and D-NPs, $n=2$ for A-NPs) by intravenous injection in the tail vein. At regular time intervals post injection, (t=35 minutes $(0.58 \mathrm{~h}), 2 \mathrm{~h}, 4 \mathrm{~h}, 6 \mathrm{~h}, 24 \mathrm{~h}$ and 48 h) mice were anesthetized with isoflurane and placed in ventral position in the IVIS Lumina ${ }^{\circledR}$. Fluorescence images were recorded as described previously (see section Fluorescence imaging settings and image analysis)

Statistical analysis. 
Statistical analysis was performed with the Prism GraphPad 7.0 software. The significance was calculated using a two-way Anova method, with a Tukey's multiple comparison post-test (black asterisks in the figure) $95 \%$ confidence interval.

\section{Supporting Information}

Supporting Information is available from the Wiley Online Library or from the author.

\section{Acknowledgements}

This work was supported by the ERC Consolidator grant BrightSens (No. 648528), the ERC Advanced grant Ternanomed (No. 249835) and the LabEX nanoSaclay grant (ANR-10LABX-0035). BA was supported by LabEx Chimie des Systèmes Complexes. The CNRS and the French Ministry of Research are also warmly acknowledged for financial support. The authors declare no conflict of interest.

Received: ((will be filled in by the editorial staff))

Revised: ((will be filled in by the editorial staff)) Published online: ((will be filled in by the editorial staff))

\section{References}

[1] a) P. Couvreur, C. Vauthier, Pharm. Res. 2006, 23, 1417; b) M. W. Tibbitt, J. E. Dahlman, R. Langer, J. Am. Chem. Soc. 2016, 138, 704.

[2] a) A. K. Lytton-Jean, K. J. Kauffman, J. C. Kaczmarek, R. Langer, Cancer. Treat. Res. 2015, 166, 293; b) C. A. Schütz, L. Juillerat-Jeanneret, H. Mueller, I. Lynch, M. Riediker, Nanomedicine 2013, 8, 449.

[3] a) S. Mura, D. T. Bui, P. Couvreur, J. Nicolas, J. Control. Release 2015, 208, 25; b) J. Rautio, H. Kumpulainen, T. Heimbach, R. Oliyai, D. Oh, T. Jarvinen, J. Savolainen, Nat. Rev. Drug Discov. 2008, 7, 255. 
[4] a) P. Couvreur, B. Stella, L. H. Reddy, H. Hillaireau, C. Dubernet, D. Desmaele, S. Lepetre-Mouelhi, F. Rocco, N. Dereuddre-Bosquet, P. Clayette, V. Rosilio, V. Marsaud, J. M. Renoir, L. Cattel, Nano Lett. 2006, 6, 2544; b) S. Harrisson, J. Nicolas, A. Maksimenko, D. T. Bui, J. Mougin, P. Couvreur, Angew. Chem. Int. Ed. Eng.l 2013, 52, 1678.

[5] a) A. Maksimenko, M. Alami, F. Zouhiri, J. D. Brion, A. Pruvost, J. Mougin, A. Hamze, T. Boissenot, O. Provot, D. Desmaele, P. Couvreur, ACS Nano 2014, 8, 2018; b) A. Maksimenko, F. Dosio, J. Mougin, A. Ferrero, S. Wack, L. H. Reddy, A. A. Weyn, E. Lepeltier, C. Bourgaux, B. Stella, L. Cattel, P. Couvreur, Proc. Natl. Acad. Sci. U. S. A. 2014, 111, E217.

[6] a) L. H. Reddy, C. Dubernet, S. L. Mouelhi, P. E. Marque, D. Desmaele, P. Couvreur, J. Control. Release 2007, 124, 20; b) L. H. Reddy, P. E. Marque, C. Dubernet, S. L. Mouelhi, D. Desmaele, P. Couvreur, J. Pharmacol. Exp. Ther. 2008, 325, 484.

[7] L. Yildirimer, N. T. Thanh, M. Loizidou, A. M. Seifalian, Nano Today 2011, 6, 585.

[8] I. Medintz, N. Hildebrandt, Eds., in FRET-Förster Resonance Energy Transfer: From Theory to Applications, 2013.

[9] a) Y. Li, M. S. Budamagunta, J. Luo, W. Xiao, J. C. Voss, K. S. Lam, ACS Nano 2012, 6, 9485; b) S. Linden, M. K. Singh, K. D. Wegner, M. Regairaz, F. Dautry, F. Treussart, N. Hildebrandt, Dalton Trans. 2015, 44, 4994.

[10] a) R. Bouchaala, L. Mercier, B. Andreiuk, Y. Mely, T. Vandamme, N. Anton, J. G. Goetz, A. S. Klymchenko, J. Control. Release 2016, 236, 57; b) A. Gaudin, O. Tagit, D. Sobot, S. Lepetre-Mouelhi, J. Mougin, T. F. Martens, K. Braeckmans, V. Nicolas, D. Desmaële, S. C. de Smedt, N. Hildebrandt, P. Couvreur, K. Andrieux, Chem. Mater. 2015, 27, 3636; c) J. Gravier, L. Sancey, S. Hirsjarvi, E. Rustique, C. Passirani, J. P. Benoit, J. L. Coll, I. Texier, Mol. Pharm. 2014, 11, 3133; d) A. S. Klymchenko, E. Roger, N. Anton, H. Anton, I. Shulov, J. Vermot, Y. Mely, T. F. Vandamme, RSC Advances 2012, 2, 11876.

[11] S. W. Morton, X. Zhao, M. A. Quadir, P. T. Hammond, Biomaterials 2014, 35, 3489.

[12] R. L. Simmons, R. T. Yu, A. G. Myers, J. Am. Chem. Soc. 2011, 133, 15870.

[13] G. Hong, A. L. Antaris, H. Dai, Nat. Biomed. Eng. 2017, 1, 0010.

[14] S. Preus, L. M. Wilhelmsson, ChemBioChem 2012, 13, 1990.

[15] L. Bildstein, C. Dubernet, V. Marsaud, H. Chacun, V. Nicolas, C. Gueutin, A. Sarasin, H. Benech, S. Lepetre-Mouelhi, D. Desmaele, P. Couvreur, J. Control. Release 2010, 147, 163. 
[16] L. C. Simon, C. M. Sabliov, Drug Metab. Rev. 2014, 46, 128.

[17] A. K. Mohammad, J. J. Reineke, Mol. Pharm. 2013, 10, 2183.

[18] a) N. Khlebtsov, L. Dykman, Chem. Soc. Rev. 2011, 40, 1647; b) Y. N. Zhang, W. Poon, A. J. Tavares, I. D. McGilvray, W. C. W. Chan, J. Control. Release 2016, 240, 332; c) L. Yang, H. Kuang, W. Zhang, Z. P. Aguilar, H. Wei, H. Xu, Sci. Rep. 2017, 7, 3303.

[19] G. Xie, J. Sun, G. Zhong, L. Shi, D. Zhang, Arch. Toxicol. 2010, 84, 183.

[20] S. K. Balasubramanian, J. Jittiwat, J. Manikandan, C. N. Ong, L. E. Yu, W. Y. Ong, Biomaterials 2010, 31, 2034.

[21] P. Lin, J. W. Chen, L. W. Chang, J. P. Wu, L. Redding, H. Chang, T. K. Yeh, C. S. Yang, M. H. Tsai, H. J. Wang, Y. C. Kuo, R. S. Yang, Environ. Sci. Technol. 2008, $42,6264$.

[22] J. Kolosnjaj-Tabi, Y. Javed, L. Lartigue, J. Volatron, D. Elgrabli, I. Marangon, G. Pugliese, B. Caron, A. Figuerola, N. Luciani, T. Pellegrino, D. Alloyeau, F. Gazeau, ACS Nano 2015, 9, 7925.

[23] A. Gaudin, M. Yemisci, H. Eroglu, S. Lepetre-Mouelhi, O. F. Turkoglu, B. DonmezDemir, S. Caban, M. F. Sargon, S. Garcia-Argote, G. Pieters, O. Loreau, B. Rousseau, O. Tagit, N. Hildebrandt, Y. Le Dantec, J. Mougin, S. Valetti, H. Chacun, V. Nicolas, D. Desmaele, K. Andrieux, Y. Capan, T. Dalkara, P. Couvreur, Nat. Nanotechnol. 2014, 9, 1054.

[24] L. C. Kennedy, L. R. Bickford, N. A. Lewinski, A. J. Coughlin, Y. Hu, E. S. Day, J. L. West, R. A. Drezek, Small 2011, 7, 169.

[25] A. Alessi, M. Salvalaggio, G. Ruzzon, J. Lumin. 2013, 134, 385.

$\phi \quad$ The observed signal at the FRET channel for D-NPs was due to some leakage of the donor dye emission through the filter used in our FRET channel (810-875 nm). 
Table of contents entry

Förster resonance energy transfer (FRET) is used, for the first time, to monitor the in vivo fate of prodrug-based nanoparticles. After the initial hepatic accumulation, nanoparticles show loss of their integrity within $2 \mathrm{~h}$ and clearance of the squalene bioconjugates is observed within $24 \mathrm{~h}$. Such awareness is a key prerequisite before introduction into clinical settings.

Keyword

FRET, prodrug nanoparticles, in vivo non-invasive imaging 
Fanny Cayre, Simona Mura* Bohdan Andreiuk, Dunja Sobot, Sandrine Gouazou, Didier Desmaele, Andrey S. Klymchenko, Patrick Couvreur*

In Vivo FRET Imaging to Predict the Risk Associated with Hepatic Accumulation of Squalene-Based Prodrug Nanoparticles

\section{ToC figure}

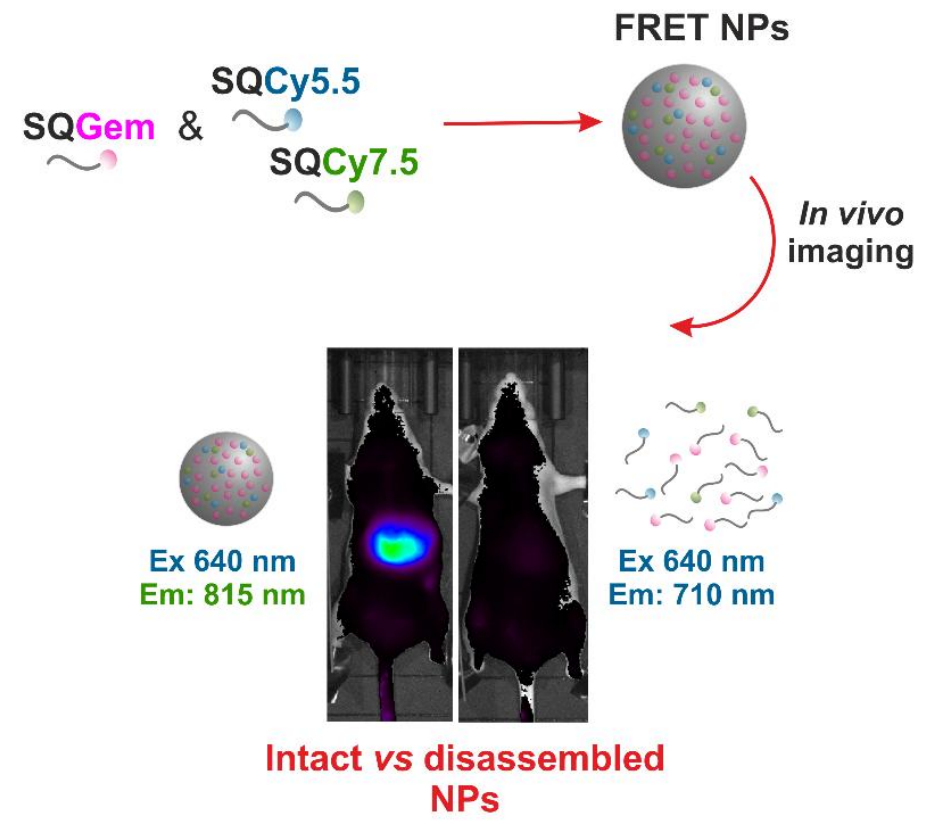

